\title{
HISTORIA DEL INSTITUTO NACIONAL DE SALUD OCUPACIONAL DEL PERÚ
}

\author{
Juan M. Cossio-Brazzan ${ }^{1, a}$
}

\begin{abstract}
RESUMEN
En el Perú, el desarrollo de la industria ha generado mejoras económicas pero, a la vez, ha tenido un gran impacto en la salud de sus trabajadores por lo que era necesario implementar mecanismos de control. Por ello, en 1940 se crea el Departamento de Higiene Industrial, que en 1956 cambia a Instituto de Salud Ocupacional, que fue desactivado en 1994. Sin embargo, el 2001 reaparece en la estructura organizacional del Ministerio de Salud como Instituto de Salud Ocupacional "Alberto Hurtado Abadía". En la actualidad, es el Centro Nacional de Salud Ocupacional y Protección del Ambiente para la Salud (CENSOPAS), órgano del Instituto Nacional de Salud que continúa trabajando en sinergia con otras instituciones y sectores, generando evidencias para proteger la salud de las personas expuestas (trabajadores y comunidad) a la contaminación y riesgos asociados con las actividades económicas.
\end{abstract}

Palabras clave: Historia; Salud ocupacional; Industria; Profesiones; Higiene industrial (fuente: DeCS BIREME).

\section{HISTORY OF INSTITUTO NACIONAL DE SALUD OCUPACIONAL DEL PERU}

\begin{abstract}
In Peru, the industry's development has made economic improvements but at the same time, it has had a major impact on the health of the workers; for that reason, it was necessary to generate control mechanisms. So, in 1940 it was created the Departmento de Higiene Industrial, which in 1956 was changed to Instituto de Salud Ocupacional, but it was deactivated in 1994. However, in 2001 it reappeared into the Ministerio de Salud organizational structure with the name of Instituto de Salud Ocupacional "Alberto Hurtado Abadía". Actually, it is the Centro Nacional de Salud Ocupacional y Protección del Ambiente para la Salud (CENSOPAS), organ of the Instituto Nacional de Salud which continues working in synergy with other institutions and sectors, making research to protect the health of exposed persons (workers and community) to contamination and risks associated with economic activities.
\end{abstract}

Key words: History; Occupational Health; Industry; Occupations; Industrial health (source: MeSH NLM).

El gran auge y crecimiento de la minería en el Perú y el incremento de la industria en las primeras décadas del siglo pasado, así como el desarrollo del derecho del trabajo y la seguridad social, hicieron evidente que se necesitaba un programa de salud ocupacional que permitiera prevenir los accidentes y enfermedades ocupacionales; el control de los riesgos ocupacionales; la contaminación ambiental; y que hiciera posible la sostenibilidad de las actividades económicas sin costos ni conflictos sociales ${ }^{(1)}$.

El 5 de agosto del año 1940 el DS 1818 crea el Departamento de Higiene Industrial, en el Ministerio de Salud Pública, Trabajo y Promoción Social, financiado desde 1947 con el aporte del $1,8 \%$ de la planilla de las empresas mineras (Ley 10833). En 1948, en base a un acuerdo entre Perú y Estados Unidos, el Servicio Interamericano de Salud Pública asume la administración del Departamento; con sus aportes y los de la Ley 10833, el personal recibe capacitación en el extranjero y se equipa con alta tecnología de la época en medicina e ingeniería ocupacional y ambiental; en laboratorio clínico, químico toxicológico y una biblioteca especializada (2). Profesionales con reconocimiento mundial como el Dr. Vintinner y el Ing. Bloomfield trabajaron varios años en el Departamento de Higiene Industrial o Instituto de Salud Ocupacional (INSO) del Perú. EI INSO fue el primero en crearse en la región andina $\mathrm{y}$, en reconocimiento de su desarrollo, fue el centro de capacitación para la región.

A partir de 1957, para ampliar su cobertura, se descentraliza el Instituto de Salud Ocupacional con la creación de tres unidades regionales ubicadas en las ciudades de La Oroya, Trujillo y Arequipa, cubriendo así las regiones del centro, norte y sur respectivamente.

Desde 1956 hasta mediados de los años setenta se público la Revista del Instituto de Salud Ocupacional. Entre los artículos publicados, podemos mencionar temas como el trabajo en altura; valores hemáticos y trabajo minero; aspectos radiográficos cardiopulmonares en el mal de montaña; presión arterial en mineros; presión arterial en trabajadores

Centro Nacional de Salud Ocupacional y Protección del Ambiente para la Salud, Instituto Nacional de Salud. Lima, Perú.

Psicólogo

Recibido: 09-05-12 Aprobado: 13-06-12 
a 4600 metros de altitud; diagnóstico de silicosis; aplicación de la cromatografía de gases a los estudios de ventilación pulmonar; estudio comparativo entre el detector ultravioleta y los métodos químicos para la determinación de vapores de mercurio en aire; entre otros. En el periodo comprendido entre el año 1956 a 1970 , se publicaron 189 trabajos, entre los que están incluidos los mencionados anteriormente ${ }^{(3)}$.

EI INSO fue reconocido en todos los sectores laborales, principalmente en el minero; prueba de ello es que las principales normas sobre Salud Ocupacional de los años cincuenta hasta los noventa lo mencionan. Por ello se le otorgó un papel relevante en ese ámbito, en reconocimiento a su elevada competencia técnica.

En el año 1991, con Resolución Ministerial 000991-SA/DM, se aprueba la Apertura Programática de Actividades del Ministerio de Salud, en ella se considera al INSO como parte del Instituto Nacional de Salud. En 1994 con RM 552-94-SA/DM es considerado fenecido, y sus recursos y funciones pasan a la Dirección General de Salud Ambiental del Ministerio de Salud, desactivándose la unidad de Lima y sus tres unidades regionales; minimizando así al límite las actividades de salud ocupacional; asimismo, se desactivó la única biblioteca especializada en salud ocupacional del Perú, lo que ocasionó la pérdida de gran parte de su material bibliográfico y de los informes técnicos sobre las visitas de reconocimiento, evaluación de riesgos, y exámenes médicos realizados. Estos informes técnicos eran de vital importancia pues permitía hacer un adecuado seguimiento a estas empresas, principalmente mineras.

Sin embargo, el 26 de julio de 2001 se publica en el diario oficial "El Peruano" la RM 413-2001-SA/DM que reintegra el Instituto Nacional de Salud Ocupacional a la estructura orgánica del Ministerio de Salud, denominándolo Instituto de Salud Ocupacional "Alberto Hurtado Abadía"; asimismo, se establece que los recursos, y todo lo que fue transferido del ex-INSO a la Dirección General de Salud Ambiental, sea transferido al Instituto de Salud Ocupacional "Alberto Hurtado Abadía",

En el 2002, la Ley del MINSA (Ley 27657) une al Instituto de Salud Ocupacional "Alberto Hurtado Abadía" con el Instituto Nacional de Protección del Ambiente para la Salud (INAPMAS) denominándose Centro Nacional de Salud Ocupacional y Protección del Ambiente para la Salud (CENSOPAS) y pasa a ser uno de los órganos técnicos del Instituto Nacional de Salud. Si bien a en Lima se viene fortaleciendo, en las regiones aún no se tiene instituciones que aborden la salud de los trabajadores, y de poblaciones expuestas, a la contaminación relacionada con las actividades económicas; queda todavía sin cubrir, lo que las ex unidades regionales de salud ocupacional realizaban ${ }^{(4)}$.

La no priorización, o poco conocimiento sobre salud ocupacional o seguridad y salud en el trabajo, ha generado que tengamos normativas dispersas $y$ con vacíos técnicos además de desconocimiento de la magnitud y distribución de estos problemas; las estadísticas sobre accidentes de trabajo tienen alto subregistro, y sobre enfermedades ocupacionales la situación es aun más crítica, pues son muy limitados los recursos humanos capacitados para realizar diagnósticos de enfermedades ocupacionales. En los últimos años el CENSOPAS viene desarrollando actividades con la Organización Internacional del Trabajo y con la Universidad Peruana Cayetano Heredia, para capacitar profesionales y técnicos, pero aún es insuficiente; por lo tanto, se requiere que las universidades participen para poder contar con los profesionales y técnicos que el país requiere. También, pese a las limitaciones, el CENSOPAS viene fortaleciéndose en tecnología capacitando a sus profesionales en el extranjero; asimismo, el marco legal actual, al aprobarse la Ley 29783 "Ley de Seguridad y Salud en el Trabajo" en agosto de 2011, y aprobarse su Reglamento el 5 de abril del presente año, entre los múltiples aspectos que aborda, designa al CENSOPAS como miembro del Consejo Nacional de Seguridad y Salud en el Trabajo; este mecanismo de concertación permitirá el desarrollo de la salud ocupacional; prevenir los accidentes y enfermedades ocupacionales que sufren los trabajadores y afectan a sus familias y a las empresas promoviendo su competitividad sostenible; a las autoridades, organizaciones laborales y empresariales, poder sumar esfuerzos para asumir en conjunto el reto de la seguridad y salud en el trabajo, y contribuir al desarrollo de nuestro Perú.

\section{REFERENCIAS BIBLIOGRÁFICAS}

1. Priale PJ. Epidemiología general, Salud Ocupacional y Ambiental. Temas escogidos. 1ra edición. Lima: Asociación Canadiense de Salud Pública; 2000.

2. Vinces A. Contaminación Ambiental y Defensa Ecológica de Lima. 1ra edición. Lima: Editora Yuracmayo E.I.R.L., 1993.

3. Rodriguez L, Ochoa R. Índice de Autores, materias y resúmenes de los trabajos publicados en los boletines y revistas del Instituto de Salud Ocupacional durante quince años (1956 - 1970). Revista del Instituto de Salud Ocupacional. 1970;XV(1-4).

4. Academia Peruana de Salud. Historia de la Salud en el Perú: Rehabilitación. Salud ocupacional. Lima: Academia Peruana de Salud; 2008.

Correspondencia: Juan M. Cossio Brazzan

Dirección: Cl. Las Amapolas 350, Urb. San Eugenio. Lima 14, Perú.

Teléfono: (511) 617-6177

Correo electrónico: jcossio@ins.gob.pe 